\title{
On the X-ray emission of the low-mass galaxy groups
}

\author{
H. M. Tovmassian and O. Yam \\ Instituto Nacional de Astrofísica Óptica y Electrónica, AP 51 y 216, 72000, Puebla, Pue, México \\ hrant@inaoep.mx, oyam@inaoep.mx \\ and \\ H. Tiersch \\ Sternwarte Königsleiten, 81477, München, Leimbachstr. 1a Germany \\ htiersch@uni.de
}

Received ...2001 / Accepted .. 2001

\begin{abstract}
It is shown that the low-mass groups obey the $L_{x} \sim \sigma_{v}^{4}$ law deduced for galaxy clusters. The impression of the more shallow slope of the $L_{x}-\sigma_{v}$ correlation for groups is created not by enhanced X-ray emission, but by underestimation of the radial velocity dispersion of some groups.

Subject headings: galaxies: clusters : general - X-rays: galaxies
\end{abstract}

\section{Introduction}

Solinger \& Tucker (1972) showed that if the source of the $\mathrm{X}$-ray radiation is hot gas bound in clusters, then the X-ray luminosity, $L_{x}$, should be correlated with the radial velocity dispersion, $\sigma_{v}$. Thermal emission from the intracluster gas yields an X-ray luminosity, $L_{x}$, proportional to the square of the gas density. In the case of a constant mass-to-light ratio, the $L_{x}$ is proportional to the square of the mass of the cluster. If the cluster is a relaxed system, $\sigma_{v}$ is roughly proportional to the square root of the mass. Thus, $L_{x} \propto \sigma_{v}^{4}$. Quintana \& Melnick (1982) showed that $L_{x}$ of galaxy clusters, indeed, obeys the expected correlation. Dell'Antonio et al. (1994) showed that rich groups follow the $L_{x} \propto \sigma_{v}^{4}$ relation, but groups with smaller $\sigma_{v}$ do have more shallow slope, $L_{x} \propto \sigma_{v}^{2.7}$. All recent observations (Mahdavi et al. 1997, Zabludoff \& Mulchaey 1998, Helsdon \& Ponman 2000, Mahdavi et al. 2000, Xue \& Wu 2000, Mahdavi et al. 1997) proved that the dependence of the X-ray emission for low-mass groups (which includes compact groups) on the $\sigma_{v}$ is much weaker than for galaxy clusters. Zimer et al. (2001) analyzing the results of different investigators mentioned some discrepancies, and concluded that they were due to poorly determined $\sigma_{v}$ s and $L_{x}$ s. All data, show, however, that some amount of low-mass groups of galaxies are located on the left side of the line $L_{x} \propto \sigma_{v}^{\sim 4}$. It has been generally assumed that the reason of such location of groups on the graph $L_{x}-\sigma_{v}$ is the enhanced (by one-two orders of magnitude) X-ray emission of groups. It is widely assumed that the excess Xray luminosity of the low-mass groups is explained by the "mixed emission" scenario (Dell'Antonio et al. 1994) when the emission from the intragroup plasma may be contaminated by a superposition of diffuse X-ray sources corresponding to the hot interstellar medium of the member galaxies. However, the shift of groups to the left of the $L_{x} \propto \sigma_{v}^{4}$ line may have another reason: underestimation of $\sigma_{v} \mathrm{~s}$.

It has been shown that HCGs and ShCGs have a triaxial spheroid, "cigar"-like shapes (Malykh \& Orlov 1986, Hickson et al. 1984, Oleak et al. 1998). In a series of papers Tovmassian and collaborators (Tovmassian et al. 1999, Tovmassian \& 
Chavushyan 2000, Tovmassian et al. 2001) showed that $\sigma_{v}$ s of compact groups (CGs), and of associated with them loose groups (LGs) which are also elongated and have the same orientation as corresponding CGs, are correlated with elongation of groups determined by $b / a$ ratio $^{1}$. It means that members of CGs and LGs move along the elongation of corresponding group. It has been shown by Tovmassian (2001a, 2001b) and Tovmassian \& Tiersch (2001) that out of three possibilities of such movement: flying out of galaxies from the center of the group in opposite directions, infalling from opposite directions, and regular rotation of member galaxies in elongated orbits around the gravitational center of each system, the latter possibility is the more realistic one. The rotation time is less than $\sim 3 \times 10^{9}$ years, so CG+LG systems may well be virialized. The measured $\sigma_{v} \mathrm{~s}$ of such elongated groups depend on the orientation of the group. The highest values of $\sigma_{v} \mathrm{~s}$ are observed in those groups orientation of elongation of which is close to the line of sight. Such groups generally have the highest $b / a$ ratio, though the chain-like groups oriented at small angles $\theta$ to the line of sight would also have relatively high $\sigma_{v}$. The measured $\sigma_{v}$ s of the majority of groups oriented at intermedient angles to the line of sight are smaller. The groups oriented close to the orthogonal to the line of sight have the smallest measured $\sigma_{v}$ s, i.e. their $\sigma_{v}$ s are highly underestimated. The latter groups are the most elongated and have the smallest $b / a$ ratio. Hence, they would be located on the left part of the $L_{x}-\sigma_{v}$ graph. We show in this paper that underestimation of $\sigma_{v} \mathrm{~s}$ of the seen edge-on elongated groups, indeed, creates the more shallow slope of the $L_{x}-\sigma_{v}$ correlation.

\section{Analysis of data}

For this analysis we used the dataset of Mahdavi et al. (2000) which is the largest sample of the low-mass galaxy groups (RASSCALS) with detected X-ray emission, and the X-ray selected sample of HCGs (Ponman et al. 1996). We compared $b / a$ ratios of the groups most remote to the left from the line $L_{x} \propto \sigma_{v}^{4}$ on the $L_{x}-\sigma_{v}$ graph

\footnotetext{
${ }^{1} a$ is the angular distance between the most widely separated galaxies in the group, and $b$ is the sum of the angular distances $b_{1}$ and $b_{2}$ of the most distant galaxies on either side of the line $a$ joining the most separated galaxies (Rood 1979).
}

with those of the most remote to the right, or with those of the all other groups. The ratios $b / a$ for RASSCALS were determined on the corresponding maps presented in Mahdavi et al. (2000), and those of for HCGs were taken from Tovmassian et al. (1998). In the latter paper it is shown that the correlation between $\sigma_{v}$ s and $b / a$ s does not depend on the number of group members.

The following ten RASSCALS: SRGb063 (0.35), SRGb075 (0.60), SRGb119 (0.44), SSb085 (0.43), NRGb045 (0.61), SS2b153 (0.52), NRGs317 (0.43), SRGb009 (0.27), SS2b293 (0.41), and SS2b313 (0.37) are the most remote to the left (Fig. 1). The groups SS2b056 (0.87), NRGb004 (0.77), NRGs027 (0.62), NRGb0032 (0.56), NRGs110 (0.50), NRGs117 (0.75), NRGb155 (0.96), NRGs388 (0.45), SS2b261 (0.46), and SRGs040 (0.87) are the most remote to the right. The corresponding $b / a$ values are listed in parentheses. The other 49 groups with measured X-ray emission are located along the $L_{x} \propto \sigma_{v}^{4}$ line within the stripe with $\log \sigma_{v}= \pm 0.1$ width. The mean $b / a$ of the groups at the left is equal to $0.44 \pm 0.11$, and that of the groups at the right is $0.68 \pm 0.19$.

The same trend is observed in the case of HCGs. The mean value of $b / a$ s of four groups located most remote to the left of the line $L_{x}-\sigma_{v}^{4}$ (Fig. 2) is equal to $0.20 \pm 0.05$, while that of the rest 18 groups is equal to $0.44 \pm 0.18$. The Kolmogorov-Smirnov test rejected the hyphotesis that the compared distributions are of the same parent distribution in both considered cases. The significance level of rejection for the RASSCALS is $\alpha=0.0069$, and for HCGs is $\alpha=0.0082$.

Hence, the groups located to the left of the $L_{x} \propto \sigma_{v}^{4}$ line on the $L_{x}-\sigma_{v}$ graph have the smallest $b / a$ ratios. It indicates, that they are oriented close to the orthogonal to the line of sight. For this reason their measured radial velocity dispersions are smaller than the real values. Location of such groups on the left side of the $L_{x} \propto \sigma_{v}^{4}$ line creates an impression of the more shallow slope of the $L_{x}-\sigma_{v}$ correlation. Possible errors in $b / a$ values caused by missed members or chance interlopers would, apparently, increase the dispersion of the mean values, but would not introduce systematic errors in either direction.

The highest $\sigma_{v}$ s of HCGs are $\sim 600 \mathrm{~km} \mathrm{~s}^{-1}$. The highest $\sigma_{v}$ s of the groups studied by Mahdavi et al. (2000) are of the same order. Apparently, 


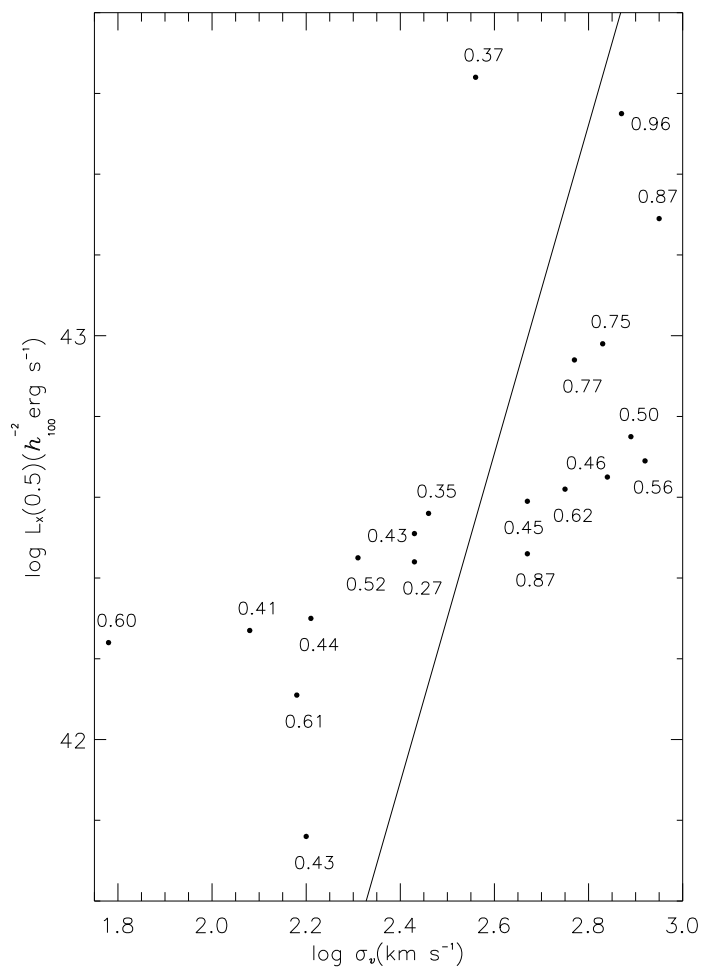

Fig. 1. $-L_{x}-\sigma_{v}$ relation of groups. Data are taken from Mahdavi et al. (2000). Only the groups located at the utmost left and the utmost right from the line $L_{x} \propto \sigma_{v}^{4}$ are drawn. The $b / a$ ratios are shown.

these are the groups observed end-on. Their measured $\sigma_{v}$ s generally correspond to the real values. Such groups would be located at the utmost right of the $L_{x} \propto \sigma_{v}$ graph. With the increase of the angle $\theta$ the $b / a$ ratio and the corresponding $\sigma_{v}$ of the group would decrease. At angles $\theta$ of about $75^{\circ}$ the measured $\sigma_{v}$ s would be by about $400 \mathrm{~km}$ $\mathrm{s}^{-1}$ smaller in comparison to the real values, and $\log \sigma_{v}$ would be about 2.2, which corresponds to that of the groups located at the utmost left on the $L_{x}-\sigma_{v}$ graph.

Thus, not the X-ray luminosities of the lowmass groups are higher than it is expected by the $L_{x} \propto \sigma_{v}^{4}$ law, but the $\sigma_{v}$ s of them are underestimated. Rich clusters have much more symmetric round shape, and orientation may have negligible effect on the measured $\sigma_{v}$. The spread of clusters around the $L_{x} \propto \sigma_{v}^{4}$ line is due to the natural dispersion of parameters.

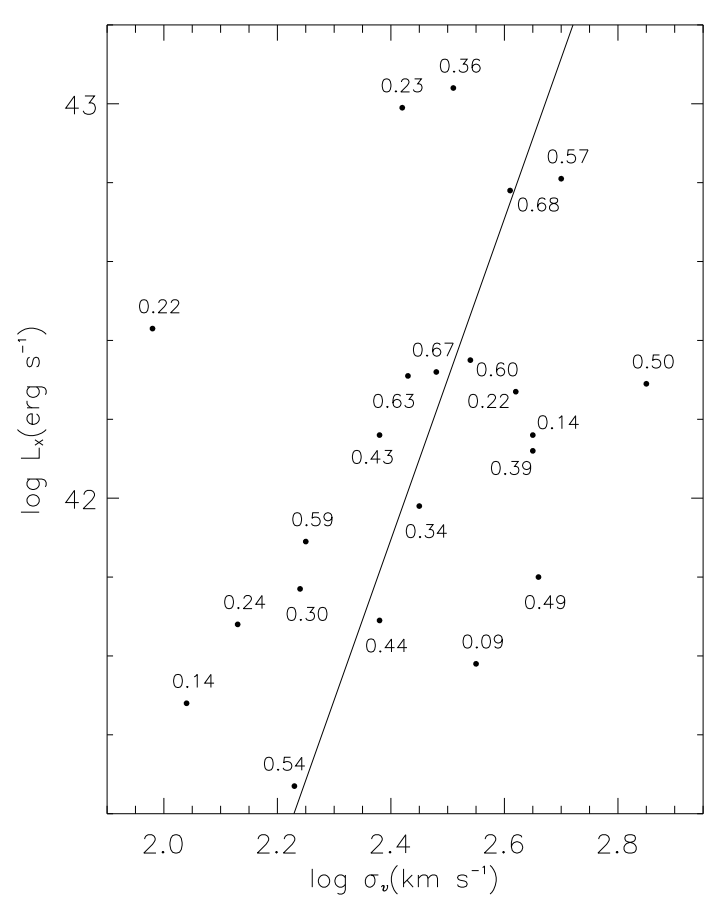

Fig. 2. $-L_{x}-\sigma_{v}$ relation for HCGs (from Ponman et al. 1996). The line $L_{x} \propto \sigma_{v}^{4}$ is drawn as in Fig. 1. The $b / a$ ratios are shown.

\section{Conclusions}

Consideration of the $b / a$ ratios of the low-mass groups shows that groups located at the utmost left of the $L_{x} \propto \sigma_{v}^{4}$ line on the $L_{x}-\sigma_{v}$ graph have, on average, smaller $b / a$ values than those located at the utmost right. The elongation of groups with small $b / a$ ratios are oriented close to the orthogonal to the line of sight. The measured $\sigma_{v}$ s of these groups are, thus, underestimated, and are smaller than the real values. Therefore, such groups are artificially shifted to the left on the $L_{x}-\sigma_{v}$ graph. This creates an impression of an enhanced X-ray luminosity. If to take into account the reasonable amount of underestimation of $\sigma_{v} \mathrm{~s}$ (of the order of 200-300 $\mathrm{km} \mathrm{s}^{-1}$, which corresponds to angle $\theta$ of about $40^{\circ}-50^{\circ}$ ), then the corresponding groups will be moved to the right, towards the line $L_{x} \propto \sigma_{v}^{4}$. Hence, the low-mass groups obey the $L_{x} \propto \sigma_{v}^{4}$ law for clusters of galaxies (Solinger \& Tucker 1972, Quintana \& Melnick 1982). It means that there is no need to apply any mechanism of the enhancement of the X-ray luminosity of the low-mass groups, since in reality there is no 
any enhancement.

Compact groups are stable systems with members probably rotating around the gravitational center of the corresponding group (Tovmassian 2001a, 2001b). For the reason of regular movement in elongated orbits the velocities of member galaxies in the central region of a CG are high enough. Therefore, the efficiency of interaction in such groups would be smaller than in the made numerical simulations when such regular movement has been neglected (Barnes 1985, 1989; Ishizawa 1986; Mamon 1987, 1990; Zheng et al. 1993). The formation of the hot interstellar medium in member galaxies, widely assumed for explanation of the excess X-ray emission, may be very rare, and may not dominate the global X-ray emission. In fact, the claimed by many excess of the X-ray luminosity in the low-mass groups is due to a projection effect, and is, thus, a result of misinterpretation of the observational data. The finding of Mahdavi \& Geller (2001) that clusters of galaxies and single elliptical galaxies form a continuous relation $L_{x}-\sigma_{v}^{m}$ are consistent with the result presented in this paper. Low-mass groups are not exotic objects and obey the same law.

We are grateful to the first Referee of our paper, Dr. A.Mahdavi, and especially to the anonymous Referee for very valuable comments which allowed us to improve the paper.

\section{REFERENCES}

Barnes, J.E., 1985, MNRAS, 215, 517

Barnes, J.E., 1989, Nature 338, 123

Dell'Antonio, I.P., Geller, M.J, \& Fabrikant, D.G., 1994, AJ, 107, 427

Helsdon, S.F., \& Ponman, T.J., 2000, MNRAS, 315,356

Hickson, P., Ninkov, Z., Huchra, P., Mamon, G.A., 1984, in Clusters and Groups of Galaxies, F. Mardirossian, G. Guiricin, M. Mezzetti (eds.), 367

Hickson P., Mendes de Oliveira C., Huchra J.P., Palumbo G. G. C. 1992, ApJ, 399, 353

Ishizawa, T., 1986, Astrophys. Space Sci., 119, 221
Mahdavi, A., Böhringer, H., Geller, M.J., \& Ramella, M., 1997, ApJ, 483, 68

Mahdavi, A., Böhringer, H, Geller, M.J., \& Ramella, M., 2000, ApJ, 534, 114

Mahdavi, A., \&, Geller, M.J., 2001, ApJ, 554, 129

Malykh, S.A., \& Orlov, V.V., 1986, Afz 24, 445

Mamon, G.A., 1986, ApJ 307, 426

Mamon, G.A., 1990, in Paired and Interacting Galaxies, IAU Coll. No. 124, p. 77

Oleak, H., Stoll, D., Tiersch, H., MacGillivray, H.T., 1998, Astron. Nachr. 319, 235

Ponman, T.J., Borner, P.D.J., Ebeling, H., \& Böhringer H., 1996, MNRAS, 283, 690

Quintana, H., Melnick, J., 1982, AJ. 87, 972

Rood, H.J., 1979, ApJ, 233, 21

Solinger, A.B., \& Tucker, W.H. 1972, ApJL, 175, L107

Tovmassian, H.M., Martinez, O., \& Tiersch, H., 1999, A\&A, 348, 693

Tovmassian, H.M., Chavushyan, V.H., 2000, AJ, 119,1687

Tovmassian, H.M., Yam, O., \& Tiersch, H., 2001, Rev. Mex. A\&A, 2001, 37, 173

Tovmassian, H.M., 2001a, PASP, 113, 543

Tovmassian, H.M., 2001b, ApJ, Submitted

Tovmassian, H.M., \& Tiersch, H., 2001, A\&A, $2001,378,740$

Xue, Y., \& Wu, X., 2000, ApJ, 538, 65

Zimer, M.E., Mulchaey, J.S., \& Zabludoff, A.I., 2001, astro-ph/0110046 v1

Zabludoff, A.I., \& Mulchaey, J.S., 1998, ApJ, 496, 39

Zheng, J., Valtonen, M.J., \& Chernin A.D., 1993, AJ, 105, 2047

This 2-column preprint was prepared with the AAS LATEX macros v5.0. 